https://helda.helsinki.fi

\title{
Neutrino mass generation and leptogenesis via pseudo-Nambu-Goldstone Higgs portal
}

\section{Alanne, Tommi}

2017-11-16

Alanne , T , Meroni , A \& Tuominen , K 2017 , ' Neutrino mass generation and leptogenesis via pseudo-Nambu-Goldstone Higgs portal ' , Physical Review D , vol. 96 , no. 9 , 095015 . https://doi.org/10.1103/P

http://hdl.handle.net/10138/228973

https://doi.org/10.1103/PhysRevD.96.095015

cc_by

publishedVersion

Downloaded from Helda, University of Helsinki institutional repository.

This is an electronic reprint of the original article.

This reprint may differ from the original in pagination and typographic detail.

Please cite the original version. 


\title{
Neutrino mass generation and leptogenesis via pseudo-Nambu-Goldstone Higgs portal
}

\author{
Tommi Alanne* \\ $C P^{3}$-Origins, University of Southern Denmark, Campusvej 55, DK-5230 Odense M, Denmark
}

Aurora Meroni ${ }^{\dagger}$ and Kimmo Tuominen

Department of Physics, University of Helsinki, and Helsinki Institute of Physics, P.O. Box 64, FI-00014 University of Helsinki, Finland

(Received 11 July 2017; published 16 November 2017)

\begin{abstract}
We consider an extension of the Standard Model with the global symmetry-breaking pattern $\mathrm{SO}(5) / \mathrm{SO}(4)$, where the Higgs boson arises as a pseudo-Nambu-Goldstone boson. The scalar content of the theory consists of a Standard-Model-like Higgs field and an extra real scalar field. The flavor sector of the model is extended by two right-handed neutrinos compatible with the observed light-neutrino phenomenology, and we find that the correct vacuum alignment determines the mass of the heavier neutrino eigenstate to be around $80 \mathrm{TeV}$. The new singlet-scalar state generates dynamically a Majorana mass term for the heavy-neutrino states. We show how the model leads to the correct baryon asymmetry of the Universe via leptogenesis in the case of two degenerate or hierarchical heavy neutrinos.
\end{abstract}

DOI: 10.1103/PhysRevD.96.095015

\section{INTRODUCTION}

After the discovery of the Higgs boson and thereby the verification of the Standard-Model-like electroweak symmetry-breaking (EWSB) pattern to very high accuracy, the neutrino sector provides one of the most prominent sources for phenomenology beyond the Standard Model (bSM). In addition to not explaining the neutrino mass and mixing patterns, the Standard Model (SM) does not contain fields which would act as dark matter (DM), and it does not allow for dynamical explanation of the baryon asymmetry of the Universe (BAU).

These issues provide motivation to explore extensions of the SM even if the present collider experiments have not revealed any direct signals of bSM physics. The absence of direct signals must be interpreted to imply that the new physics occurs either at scales beyond the current experimental reach and/or is very weakly coupled with the SM.

Here we contemplate the idea that the EWSB and neutrino mass generation are connected via a minimal extension of the SM scalar sector, and the neutrino sector, in turn, could mend the SM shortcomings in explaining the BAU and DM abundance. In this paper, we focus on the details of the BAU and comment on the possible routes towards DM model building.

An attractive explanation of the Majorana nature of massive neutrinos is provided by the seesaw mechanism [1-6], which not only gives an explanation of the smallness of neutrino masses via heavier fermionic singlets, but

\footnotetext{
*alanne@cp3-origins.net aurora.meroni@helsinki.fi

kimmo.i.tuominen@helsinki.fi
}

also gives an explanation to the observed BAU through leptogenesis $[7,8]$.

Connecting the neutrino mass generation to EWSB and the details of flavor physics likely requires a larger scalar sector than the SM one. The compatibility with the spectrum observed at the LHC can be achieved in models predicting the Higgs as a pseudo-Nambu-Goldstone boson (pNGB); see e.g. Ref. [9] for a review of this type of model. The Higgs sector can be either elementary or composite. While the composite case is attractive as it allows us to address the hierarchy problem, it lacks simple dynamics to produce the SM-fermion masses. The elementary case, on the other hand, provides a calculable framework to assess the observed symmetrybreaking pattern and low-energy spectrum [10], and facilitates an effective description of flavor physics in terms of Yukawa interactions [11] as in the SM. We will follow the latter route in this paper.

We will investigate the $\mathrm{SO}(5) \rightarrow \mathrm{SO}(4)$ pattern of global symmetry breaking as a renormalizable field theory simultaneously protecting the Higgs mass and reproducing a Majorana neutrino mass term. The coset of the global symmetry-breaking pattern, $\mathrm{SO}(5) / \mathrm{SO}(4)$, allows the presence of a SM scalar doublet using the fundamental representation for the scalar sector. Explicitly, the fundamental of $\mathrm{SO}(5)$ decomposes as $\mathbf{5}=\mathbf{1} \oplus(\mathbf{2}, \mathbf{2})$, and therefore a scalar singlet appears in the model. The SM extension featuring a scalar singlet (also dubbed SSM) is a common renormalizable augmentation of the Higgs sector and has been scrutinized in the literature; see e.g. Refs. [12-20]. Our model differs essentially from these models, since in our case the singlet exists as a part of a larger multiplet due to a larger global symmetry. 
We extend the $\mathrm{SO}(5) / \mathrm{SO}(4)$ model by adding two righthanded (RH) neutrinos whose interaction and mixing patterns are chosen to conform with experimental results. We will find that the presence of these fields will orient the vacuum so that a proper EWSB pattern ensues. This, together with properties of the vacuum, provides nontrivial constraints on the model parameters. Finally, the new matter fields will allow observed BAU to be generated via leptogenesis. The details of the mechanism are sensitive to whether the masses of the RH neutrinos are hierarchical or almost degenerate.

We find that the observed BAU, together with a correct pattern of EW symmetry breaking with a spectrum compatible with observations, is achieved in the following scenario: the $\mathrm{SO}(5)$ symmetry breaks at a scale of the order of $v \sim 10^{4} \mathrm{TeV}$, and the heaviest neutrino mass eigenstate has a mass around $80 \mathrm{TeV}$. The Higgs boson with mass $125 \mathrm{GeV}$ is mostly a Goldstone-like state, and the heavier singlet state in the $\mathrm{SO}(5)$ multiplet is assumed to be lighter than the heaviest RH neutrino. This forces the scalar selfcoupling to be tiny, of order $10^{-8}$. Consequently, the heavier scalar state has mass $m^{2} \sim \lambda v^{2} \sim \mathcal{O}\left(1 \mathrm{TeV}^{2}\right)$, and the trilinear Higgs coupling will be of the order of $\mathcal{O}\left(10^{-4} \mathrm{TeV}\right)$.

The paper is organized as follows. In Sec. II we outline the details of the model. In Sec. III we analyze vacuum structure and how this constrains the parameter space of the model. In Sec. IV we discuss how the observed BAU can be produced in this model. We analyze quantitatively the cases of degenerate or hierarchical neutrinos. In Sec. V we present our conclusions and outlook for further work.

\section{THE MODEL}

In this work we consider the minimal extension of the SM scalar sector incorporating an elementary pNGB Higgs. We adopt a tree-level scalar potential that features a global symmetry-breaking pattern $\mathrm{SO}(5) \rightarrow \mathrm{SO}(4)$. The scalar degrees of freedom are conveniently parametrized by a multiplet $\Sigma$ describing a linear $\sigma$-model based on the coset $\mathrm{SO}(5) / \mathrm{SO}(4)$. We adopt the following notation,

$$
\Sigma=\left(\sigma, \Pi_{1}, \Pi_{2}, \Pi_{3}, \Pi_{4}\right),
$$

where $\sigma$ is a massive scalar degree of freedom and $\Pi_{i}$, $i=1, \ldots, 4$ are the four Nambu-Goldstone (NGB) fields associated with the broken generators of $\mathrm{SO}(5)$. The general $\mathrm{SO}(5)$-symmetric potential is given by

$$
V=\frac{1}{2} m_{\Sigma}^{2} \Sigma^{T} \Sigma+\frac{\lambda}{4 !}\left(\Sigma^{T} \Sigma\right)^{2} .
$$

The electroweak (EW) gauge group, $\mathrm{SU}(2)_{\mathrm{L}} \times \mathrm{U}(1)_{Y}$, is embedded in $\mathrm{SO}(5)$ such that the three broken generators of $\mathrm{SU}(2)_{\mathrm{L}}$ are those associated with the three NGB's $\Pi_{i}$, $i=1, \ldots, 3$. The field $\Sigma$ contains an EW Higgs doublet, $H$, and another real singlet, $\varphi$, which are the EW interaction eigenstates. The relevant physical mass eigenstates will in general be mixtures of the neutral component of $H$ and $\varphi$ such that the Higgs is mostly the NGB-like state, while the heavier singlet neutral scalar is mostly the $\sigma$-like eigenstate.

Explicitly,

$$
H=\frac{1}{\sqrt{2}}\left(\begin{array}{c}
\Pi_{1}+\mathrm{i} \Pi_{2} \\
h+\mathrm{i} \Pi_{3}
\end{array}\right)
$$

and in the unitary gauge we can write the potential of Eq. (2) in terms of the neutral components of the EW eigenstates, $h=\cos \theta \Pi_{4}+\sin \theta \sigma$ and $\varphi=-\sin \theta \Pi_{4}+\cos \theta \sigma$,

$V_{0}=\frac{m_{H}^{2}}{2} h^{2}+\frac{m_{\varphi}^{2}}{2} \varphi^{2}+\frac{1}{4 !} \lambda_{H} h^{4}+\frac{\lambda_{\varphi}}{4 !} \varphi^{4}+\frac{\lambda_{H \varphi}}{12} h^{2} \varphi^{2}$,

with $m_{H}^{2}\left(\mu_{0}\right)=m_{\varphi}^{2}\left(\mu_{0}\right) \equiv m_{\Sigma}^{2}, \lambda_{H}\left(\mu_{0}\right)=\lambda_{\varphi}\left(\mu_{0}\right)=\lambda_{H \varphi}\left(\mu_{0}\right) \equiv \lambda$, where the scale $\mu_{0}$ is of the order of the symmetry-breaking scale and is determined by renormalization conditions. ${ }^{1}$

The radiative symmetry-breaking dynamics implies

$$
\langle h\rangle=v \sin \theta \equiv v_{\mathrm{w}}=246 \mathrm{GeV},
$$

and

$$
\langle\varphi\rangle=v \cos \theta .
$$

Radiative corrections single out a value for the angle $\theta$, and the large hierarchy $v_{\mathrm{w}} \ll v$ is reflected in $\sin \theta \ll 1$.

Furthermore, we introduce two RH Majorana neutrinos. We couple them via Yukawa interactions to the EW-singlet scalar state, $\varphi$, such that the Majorana masses for the RH neutrinos are induced via the symmetry breaking. However, a generic Majorana mass term for RH neutrinos is allowed by gauge interactions, and hence the most generic Lagrangian we can write is

$$
\begin{aligned}
-\mathcal{L}^{\nu}= & Y_{i j} \overline{N_{R i}}(L \tilde{H})_{j}+\frac{1}{2} \overline{\left(N_{R}\right)^{c}}{ }_{i} \mathcal{M}_{i j} N_{R j} \\
& +\frac{1}{2} \alpha_{i j} \overline{\left(N_{R}\right)^{c}}{ }_{i} N_{R j} \varphi+\text { H.c. } \\
= & M_{D} \overline{N_{R}} \nu_{L}+\frac{1}{2} \overline{\left(N_{R}\right)^{c}} M_{N} N_{R}+\text { H.c. }
\end{aligned}
$$

where

$$
M_{D}=v_{\mathrm{w}} Y \quad \text { and } \quad M_{N}=\mathcal{M}+\langle\varphi\rangle \alpha .
$$

We note that $M_{N}$ must be symmetric due to the Majorana condition $\left(N_{R}\right)^{c}=C{\overline{N_{R}}}^{T}$. The Lagrangian in Eq. (7) is a

\footnotetext{
${ }^{1}$ Note that one can relate the masses and couplings of different field components to the corresponding parameters in the $\mathrm{SO}(5)$-invariant Lagrangian only at the scale where $\mathrm{SO}(5)$ becomes effectively restored as the couplings will run differently.
} 
realization of the type I seesaw. It is always possible to diagonalize $M_{N}$; i.e. there exists a matrix $V$ such that

$$
\mathcal{M}+\langle\varphi\rangle \alpha=V D_{N} V^{T},
$$

with the heavy-neutrino mass matrix defined as $\left(D_{N}\right)_{i j} \equiv$ $m_{N i} \delta_{i j}$.

\section{VACUUM ALIGNMENT ANALYSIS}

In the following, we assume a simplified structure of the bare Majorana mass matrix $\mathcal{M}=\operatorname{diag}\left(M_{0}, M_{0}\right)$, and consider two specific textures for $\alpha$ : diagonal and almost democratic,

$$
\alpha_{0}\left(\begin{array}{cc}
1-\epsilon & 0 \\
0 & 1
\end{array}\right), \quad \alpha_{0}\left(\begin{array}{cc}
1 & 1-\epsilon \\
1-\epsilon & 1
\end{array}\right),
$$

where $\epsilon \ll 1$ is introduced to provide small splitting of masses in the diagonal case and to provide a nonzero mass contribution for the lightest eigenstate in the democratic case. In these two cases, the masses of the heavier mass eigenstate are, respectively, $M_{0}+\left|\alpha_{0}\right| v \cos \theta$ and $M_{0}+2\left|\alpha_{0}\right| v \cos \theta$, up to corrections of $\mathcal{O}(\epsilon)$. Next we consider the constraints arising from the vacuum alignment on the parameters $M_{0}$ and $\alpha_{0}$. We will find that vacuum alignment is mainly determined by $\alpha_{0}$, while the correct value of the Higgs boson mass imposes an upper limit for $M_{0}$.

As shown in Ref. [11], in the absence of the RH-neutrino sector, the EW gauge and top corrections prefer an unbroken EW symmetry, i.e. the value $\theta=0$. Switching on the coupling $\alpha_{i j}$ between the RH neutrinos and the singlet $\varphi$, the picture changes, implying a nontrivial alignment angle, $\theta$. This is due to the different dependence on the vacuum alignment angle of the RH neutrinos and the SM sector, as can be seen from Eqs. (5) and (6).

To illustrate the main features of the vacuum structure in the model under study, we first consider the simplest case setting $M_{0}=0$ in Eq. (8) and $\epsilon=0$ in Eq. (10). For the democratic $\alpha$-matrix, this corresponds to one heavy neutrino since there are two eigenvalues, one of which is zero. For the diagonal $\alpha$-matrix, this corresponds to two degenerate heavy neutrinos.

With the choice $M_{0}=0$, one can obtain simple analytic expressions. Moreover, the result on the alignment angle $\theta$ is essentially not affected by nonzero values of $M_{0}$. This is due to the fact that allowing nonzero $M_{0}$ does not alter the $\theta$-dependence of the neutrino contribution to the one-loop effective potential.

To arrive at a simple analytic expression for the nontrivial solution for the vacuum alignment angle, we also ignore the one-loop corrections from the scalar sector. In the numerical analysis, we include all these corrections and show that the results for the alignment do not essentially change from the simplified results. In this simplified limit, the effective potential up to the one-loop level can be written as

$$
V_{\text {eff }}=V_{0}+V_{1}^{\mathrm{SM}}+V_{1}^{\nu},
$$

where following the notations of Ref. [11], the one-loop contributions in the $\sigma$-background and in the $\overline{\mathrm{MS}}$ scheme can be written as

$$
\begin{aligned}
V_{1}^{\mathrm{SM}} & =\frac{3 g^{4} \sigma^{4} \sin ^{4} \theta}{64 \pi^{2}} \cdot\left(A_{\mathrm{SM}} \log \frac{g^{2} \sigma^{2} \sin ^{2} \theta}{\mu_{0}^{2}}+B_{\mathrm{SM}}\right) \\
V_{1}^{\nu} & =\frac{\left|\alpha_{0}\right|^{4}}{32 \pi^{2}} \sigma^{4} \cos ^{4} \theta \cdot\left(A_{\nu} \log \frac{\left|\alpha_{0}\right|^{2} \sigma^{2} \cos ^{2} \theta}{\mu_{0}^{2}}+B_{\nu}\right)
\end{aligned}
$$

where we define

$$
\begin{aligned}
A_{\mathrm{SM}}= & \frac{1}{16}\left[\left(\frac{g^{2}+g^{\prime 2}}{g^{2}}\right)^{2}+2\right]-\frac{y_{t}^{4}}{g^{4}}, \\
B_{\mathrm{SM}}= & \frac{1}{16}\left[\frac{8 y_{t}^{4}}{g^{4}}\left(3-\log \frac{y_{t}^{4}}{4 g^{4}}\right)-\frac{5}{3}-\log 16\right. \\
& \left.+\left(\frac{g^{2}+g^{\prime 2}}{g^{2}}\right)^{2}\left(\log \frac{g^{2}+g^{\prime 2}}{4 g^{2}}-\frac{5}{6}\right)\right], \\
A_{\nu}^{\text {diag }}= & -2, \quad A_{\nu}^{\text {democ }}=-16, \\
B_{\nu}^{\text {diag }}= & 3, \quad B_{\nu}^{\text {democ }}=-16\left(2 \log 2-\frac{3}{2}\right) .
\end{aligned}
$$

We choose the renormalization scale, $\mu_{0}$, such that the tree-level vacuum expectation value, $\langle\sigma\rangle=v$, is not changed by the one-loop corrections and determine the preferred value of the vacuum alignment by minimizing the effective potential with respect to $\theta$. This yields the following nontrivial solution for $\theta$ :

$$
\tan ^{2} \theta=-\frac{2 A_{\nu}\left|\alpha_{0}\right|^{4}}{3 A_{\mathrm{SM}} g^{4}}=\frac{512\left|\alpha_{0}\right|^{4}}{3\left(16 y_{t}^{4}-3 g^{4}-2 g^{2} g^{\prime 2}-g^{\prime 4}\right)} .
$$

We assume the SM-like RG running of the gauge and topYukawa couplings up to the renormalization scale, $\mu_{0}$.

In this simplified limit, the $\overline{\mathrm{MS}}$ mass of the Higgs can be written as

$$
\begin{aligned}
\left(m_{h}^{\overline{\mathrm{MS}}}\right)^{2}= & \frac{3 g^{4} v_{\mathrm{w}}^{2}}{16 \pi^{2} A_{\nu}\left(2 A_{\nu}\left|\alpha_{0}\right|^{4}-3 A_{\mathrm{SM}} g^{4}\right)} \\
& \times\left[-3 A_{\mathrm{SM}} g^{4}\left(2 A_{\mathrm{SM}} A_{\nu}-3 A_{\mathrm{SM}} B_{\nu}+3 A_{\nu} B_{\mathrm{SM}}\right)\right. \\
& +2 A_{\nu}\left|\alpha_{0}\right|^{4}\left(2 A_{\mathrm{SM}} A_{\nu}+3 A_{\mathrm{SM}} B_{\nu}-3 A_{\nu} B_{\mathrm{SM}}\right) \\
& \left.+3 A_{\mathrm{SM}} A_{\nu}\left(3 A_{\mathrm{SM}} g^{4}+2 A_{\nu}\left|\alpha_{0}\right|^{4}\right) \log \left(-\frac{3 A_{\mathrm{SM}} g^{2}}{2 A_{\nu}\left|\alpha_{0}\right|^{2}}\right)\right] .
\end{aligned}
$$




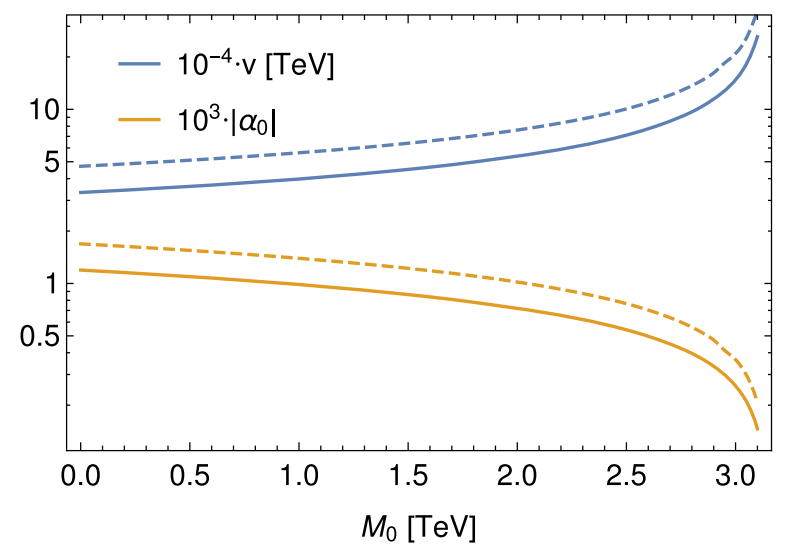

FIG. 1. The values of the vacuum expectation value and the (absolute value of the) Majorana Yukawa coupling, $\left|\alpha_{0}\right|$, as a function of $M_{0}$, when the correct Higgs mass and the vacuum solution are imposed. Solid (dashed) lines correspond to the democratic (diagonal) $\alpha$-matrices in Eq. (10).

Once we have the expression for the $\overline{\mathrm{MS}}$ mass, we calculate the physical mass following Refs. [21,22].

Next, we turn to the full numerical analysis including the one-loop corrections from the scalar sector and adding a nonzero Majorana mass parameter, $M_{0}$. The values for the symmetry-breaking scale and the absolute value of the Majorana-neutrino Yukawa coupling, $\left|\alpha_{0}\right|$, for a stable minimum solution producing the correct Higgs mass are given in Fig. 1. We find that increasing the hard Majorana mass parameter $M_{0}$, while requiring a stable minimum with respect to the angle $\theta$, constrains the minimum allowed physical mass of the Higgs. We show this dependence in Fig. 2.

The democratic or diagonal $\alpha$-matrix implies two hierarchically separated or two degenerate mass eigenvalues and, therefore, corresponds respectively to one or two heavy RH flavors. In Fig. 3 we show how the value of the

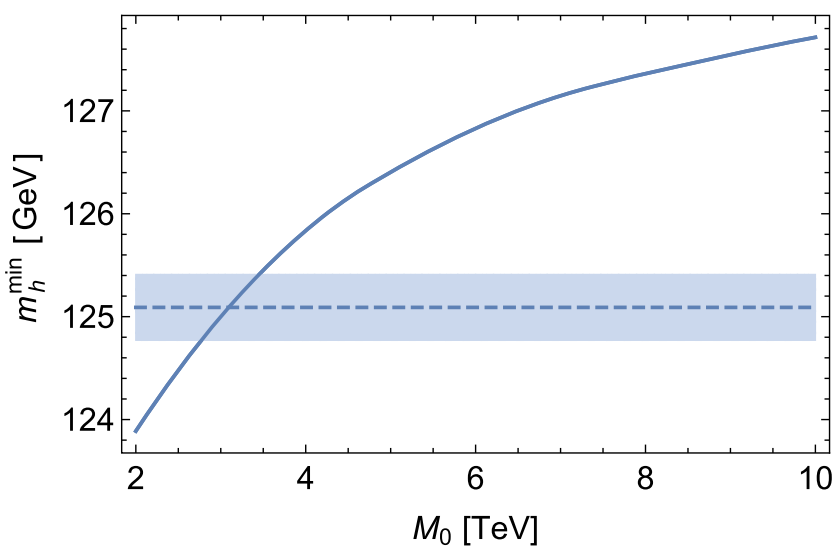

FIG. 2. The minimum allowed physical Higgs mass value as a function of $M_{0}$ when the vacuum solution is imposed. The figure corresponds to the democratic texture in Eq. (10). The result of the diagonal case is practically indistinguishable.

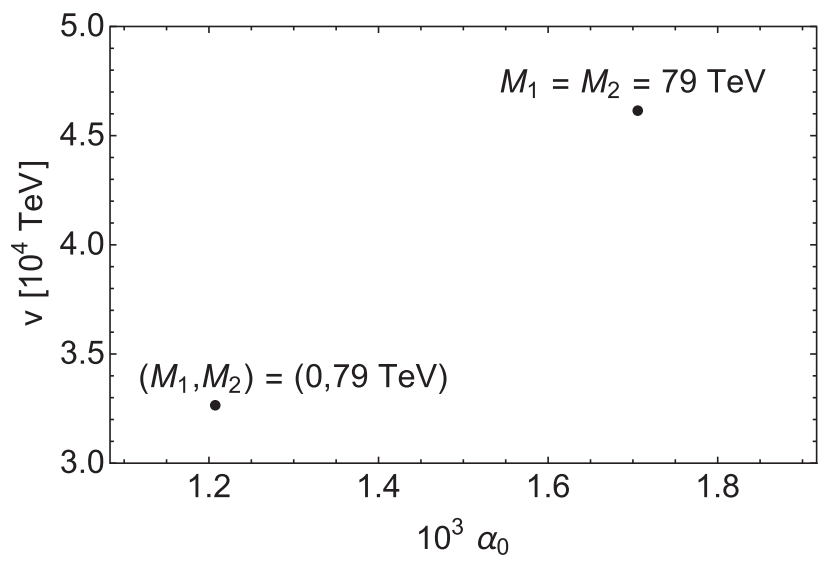

FIG. 3. The values for the symmetry-breaking scale as a function of the Majorana neutrino Yukawa coupling for one and two heavy RH neutrinos with identical couplings. The points correspond to values at $M_{0}=0$ in Fig. 1.

symmetry-breaking scale and the value of the Majorana Yukawa coupling depend on these two possibilities for the RH-neutrino mass textures. We have fixed $m_{h}^{\overline{\mathrm{MS}}}=$ $128 \mathrm{GeV}$ to account for the correct physical Higgs mass. We note that in both cases the value of the heaviest neutrino turns out to be around $80 \mathrm{TeV}$.

\section{SEESAW MECHANISM AND LEPTOGENESIS}

Baryogenesis via leptogenesis [8] is a simple mechanism to explain the BAU. The asymmetry is usually expressed in terms of the net baryon-to-photon ratio [23]

$$
\eta_{B}=\frac{n_{B}-n_{\bar{B}}}{n_{\gamma}} \simeq(6.08 \pm 0.04) \times 10^{-10} .
$$

The effect of nonperturbative $(B+L)$-violating sphaleron processes can convert a lepton asymmetry into a baryon asymmetry $[7,8]$. This scenario can be well implemented within the seesaw (type I) mechanism [1-4]: in the thermal leptogenesis scenario, after the inflation period, the heavy RH neutrinos are produced by thermal scatterings, and subsequently they can decay out-of-equilibrium, producing both lepton and $C P$ number violation, therefore satisfying all of Sakharov's conditions (see Ref. [24] for a review).

In this section, we study the different possibilities to produce the observed amount of the BAU within the model presented in the previous section. We restrict ourselves to the minimal RH-neutrino sector with two heavy flavors to illustrate the main features of generating BAU. We note, however, that extending the neutrino sector with a third $\mathrm{RH}$ neutrino would allow for a DM candidate like the one considered e.g. in Refs. [25-27]. Furthermore, the addition of a third, lighter RH neutrino would not affect the vacuum structure, since this is predominantly determined by the 
heaviest state. We leave, however, the study of DM phenomenology to future work, and concentrate here on details of how to generate the BAU.

Interestingly the two simple forms of $\alpha$ given in Eq. (10) imply different possibilities for the production of the baryon asymmetry in this model: the diagonal form will allow for resonant leptogenesis, while the democratic one will allow for the nonresonant standard scenario.

We express the neutrino Dirac-Yukawa couplings through the Casas-Ibarra parametrization [28]. We notice that this parametrization is valid also in presence of additional contributions to the bare Majorana-neutrino mass term. Indeed, in a generic basis for the heavy Majorana-neutrino mass matrix, $M_{N}$, we write

$$
M_{D}=i U_{\mathrm{PMNS}}^{*} \sqrt{D_{\nu}} \Omega \sqrt{D_{N}} V^{\dagger},
$$

with the diagonal heavy- and light-neutrino mass matrices defined respectively as $\left(D_{N}\right)_{i j} \equiv m_{N i} \delta_{i j}$ and $\left(D_{\nu}\right)_{i j} \equiv$ $m_{i} \delta_{i j}$, with $m_{i}<1 \mathrm{eV}$ and $i=1,2,3$.

The matrix $U_{\text {PMNS }}$ is the Pontecorvo-Maki-NakagawaSakata (PMNS) matrix describing leptonic interactions, $\Omega$ is a general $3 \times 3$ complex matrix that can be parametrized as $\Omega=R\left(\theta_{23}\right) R\left(\theta_{13}\right) R\left(\theta_{12}\right)$ with $\theta_{i j}$ complex, and $V$ is the matrix diagonalizing the RH Majorana mass matrix. Similarly to the standard type I seesaw scenario, one can go to the basis where the RH Majorana mass matrix is diagonal; i.e. $V$ is set to unity.

In the analysis that follows, we will restrict ourselves to the minimal case of two heavy RH neutrinos,

$$
M_{N}=\operatorname{diag}\left(M_{1}, M_{2}\right),
$$

and parametrize two different regimes in terms of $\delta \equiv$ $\left(M_{2}-M_{1}\right) / M_{1}$ :

(i) degenerate masses for $\delta \ll 1$ and

(ii) hierarchical spectrum for $\delta \gg 1$,

corresponding to our choice of a diagonal or a democratic $\alpha$-matrix, respectively. Producing the correct EW spectrum and the Higgs mass fixes the mass of the heaviest RH neutrino to be not heavier than about $80 \mathrm{TeV}$. Therefore, the simplest hierarchical leptogenesis scenario is not possible due to the Davidson-Ibarra bound [29] on the lightest $\mathrm{RH}$ neutrino mass. A possible solution to circumvent this bound and achieve a viable low-energy leptogenesis scenario is to consider nearly degenerate $\mathrm{RH}$-neutrino masses, the so-called resonant leptogenesis [30,31]. We will consider this scenario in Sec. IVA.

An alternative low-energy scenario considered in Ref. [32] is the case where the couplings between the $\mathrm{RH}$ neutrinos and the singlet scalar provide an additional source of $C P$ violation, and allow us to avoid the DavidsonIbarra bound. In this case both the bare Majorana mass and the off-diagonal mass term, $\alpha_{i j}, i \neq j$, are needed to produce a successful baryogenesis scenario. We consider a minimal extension of the model to allow for this leptogenesis scenario in Sec. IV B.

\section{A. Scenario I: Resonant leptogenesis}

Here we consider the case where the two RH neutrinos are quasidegenerate, and the oscillations between different neutrino flavors provide a sufficient enhancement of $C P$ violation. This scenario is dubbed resonant leptogenesis $[30,31]$, and it has been extensively studied in connection to the Dirac and/or Majorana $C P$-violating phases in the PMNS neutrino mixing matrix [33]. For recent developments, see e.g. Refs. [34-36]. This scenario is particularly interesting since it can directly relate the low-energy $C P$ violation in the lepton sector to the BAU.

To estimate the asymmetry, we follow Refs. [27,33,37], and the corresponding details about the resonant leptogenesis scenario can be found in Appendix A 1. We consider two heavy RH neutrinos with masses $M_{1}$ and $M_{2}$ which, in the notation of Eq. (7), satisfy

$$
\delta \equiv\left(M_{2}-M_{1}\right) / M_{1} \gg \frac{\left(Y^{\dagger} Y\right)_{12}}{16 \pi^{2}} .
$$

We assume that the baryon-to-photon ratio at recombination satisfies $\eta_{B} \simeq 0.01 N_{B-L}^{f}$, where $N_{B-L}^{f}$ is explicitly given in Eq. (A1). The proportionality constant contains the sphaleron conversion factor, $a_{\mathrm{sph}}$, which for SM particle content is $28 / 79 \sim 1 / 3$, and the dilution due to the photon production between the leptogenesis scale and the scale of recombination, $1 / f=86 / 2387 \sim 1 / 30$, assuming the standard isentropic expansion [38].

The end result of the analysis for the normal hierarchy of the active neutrinos is

$$
\delta \simeq 0.8 \times 10^{-7}\left(\frac{f\left(m_{\nu}, \Omega\right)}{f_{\max }}\right)\left(\frac{M_{1}}{10^{4} \mathrm{GeV}}\right),
$$

where $f_{\max } \simeq 0.005$, and the function $f$, defined explicitly in Appendix A 1, incorporates the dependence on the light-neutrino masses, $m_{\nu}$, and mixings via $\Omega$. With $\mathrm{RH}$ neutrinos at the scale $M_{1} \sim 80 \mathrm{TeV}$, the degeneracy of the order of $\delta \lesssim 10^{-7}$ is expected for a viable leptogenesis scenario.

Hence, this scenario requires considerable fine-tuning in the mass spectrum, but nevertheless provides a viable scenario to produce the observed BAU.

\section{B. Scenario II: Hierarchical masses}

Let us now consider leptogenesis in the case where the RH neutrinos of the model have hierarchical masses, i.e. $\delta=\left(M_{2}-M_{1}\right) / M_{1} \gg 1$. Our model contains the same degrees of freedom as the one considered in detail in Ref. [32]. We therefore follow their prescription to implement the two-stage Boltzmann equations, taking the interactions of the singlet scalar into account. The relevant 
processes for the Boltzmann equations are $\mathcal{O}\left(Y^{2}\right), \mathcal{O}\left(Y^{4}\right)$, and $\mathcal{O}\left(Y^{2} \alpha^{2}\right)$ corresponding to $\Delta L=1$ decays, $\Delta L=2$ scatterings, and $\Delta L=1$ scatterings, respectively.

The additional $C P$ violation due to the extra scalar is generated by $N_{2} \rightarrow L H$ decays via $N_{1} N_{2} \varphi$ interactions. The lighter neutrino, $N_{1}$, remains in equilibrium and can mediate potentially dangerous washout processes. However, if $N_{1}$ is weakly enough coupled, then this washout effect is suppressed, and does not disturb the enhancement of the $C P$ violation [32].

The coupled equations for the abundances of the two neutrino flavours, $Y_{1}$ and $Y_{2}$, and the lepton asymmetry $Y_{L-\bar{L}}$ are

$$
\begin{aligned}
z_{1} \frac{\partial Y_{L-\bar{L}}}{\partial z_{1}}= & \epsilon_{1} D_{1}\left(\frac{Y_{1}}{Y_{1}^{\mathrm{eq}}}-1\right)+\epsilon_{2} D_{2}\left(\frac{Y_{2}}{Y_{2}^{\mathrm{eq}}}-1\right) \\
& -Y_{L-\bar{L}}\left(W_{1 D_{1}}+W_{S_{1}}+W_{1 D_{2}}+W_{S_{2}}\right) \\
z_{1} \frac{\partial Y_{1}}{\partial z_{1}}=- & \left(\frac{Y_{1}}{Y_{1}^{\mathrm{eq}}}-1\right)\left(D_{1}+D_{21}+S_{1}\right)+\left(\frac{Y_{2}}{Y_{2}^{\mathrm{eq}}}-1\right) D_{21} \\
& -\left(\frac{Y_{1} Y_{2}}{Y_{1}^{\mathrm{eq}} Y_{2}^{\mathrm{eq}}}-1\right) S_{N_{1} N_{2} \rightarrow H H} \\
& -\left(\frac{Y_{1}^{2}}{Y_{1}^{\mathrm{eq}} 2}-1\right) S_{N_{1} N_{1} \rightarrow H H}, \\
z_{1} \frac{\partial Y_{2}}{\partial z_{1}}= & -\left(\frac{Y_{2}}{Y_{2}^{\mathrm{eq}}}-1\right)\left(D_{2}+D_{21}+S_{2}\right)+\left(\frac{Y_{1}}{Y_{1}^{\mathrm{eq}}}-1\right) D_{21} \\
& -\left(\frac{Y_{1} Y_{2}}{Y_{1}^{\mathrm{eq}} Y_{2}^{\mathrm{eq}}}-1\right) S_{N_{1} N_{2} \rightarrow H H} \\
& -\left(\frac{Y_{2}^{2}}{Y_{2}^{\mathrm{eq} 2}}-1\right) S_{N_{2} N_{2} \rightarrow H H},
\end{aligned}
$$

where $Y_{i}=n_{i} / n_{\gamma}^{\mathrm{eq}}, z_{i} \equiv M_{i} / T, z_{2}=z_{1} M_{2} / M_{1}$, and

$$
Y_{i}^{\mathrm{eq}} \equiv \frac{3}{8} z_{i}^{2} K_{2}\left(z_{i}\right)
$$

Here $K_{2}(z)$ is the modified Bessel function of the second kind. The $C P$ violation is parametrized by

$$
\epsilon_{i} \equiv \frac{\Gamma\left(N_{i} \rightarrow L H\right)-\Gamma\left(N_{i} \rightarrow \bar{L} \bar{H}\right)}{\Gamma\left(N_{i} \rightarrow L H\right)+\Gamma\left(N_{i} \rightarrow \bar{L} \bar{H}\right)},
$$

and the functions denoted by $\Gamma, D, S$, and $W$ correspond to different decay, scattering, and washout processes. The functions $D, S$, and $W$ are defined in Appendix A 2, while the decay rates, $\Gamma$, are defined in Appendix B 1 .

We can now directly proceed to presenting the numerical results we obtain for our model. The scalar sector has three parameters, $m_{\Sigma}, \lambda$ and the angle $\theta$, while the leptonic sector depends on $\alpha_{0}, \epsilon, M_{0}$ and the Yukawa matrix $Y$. The constraints on vacuum alignment and correct mass for the Higgs boson leave $M_{0}, \epsilon, Y$, and $\lambda$ as free parameters. The Yukawa matrix, $Y$, enters through the decay rates $\Gamma_{i}=\Gamma\left(N_{i} \rightarrow L H\right)+\Gamma\left(N_{i} \rightarrow \bar{L} \bar{H}\right)$ and $\epsilon$ through $\Gamma_{21}=\Gamma\left(N_{2} \rightarrow N_{1} \varphi\right)$ given explicitly in Eq. (B2). A convenient parametrization is given by considering the equilibrium parameters $K_{i}, i=1,2$, and $K_{12}$,

$$
K_{i} \equiv \frac{\Gamma_{i}}{H_{i}}=\frac{\tilde{m}_{i}}{m_{\star}}, \quad K_{21} \equiv \frac{\Gamma_{21}}{H_{2}},
$$

and we define

$$
\tilde{m}_{i} \equiv \frac{\left(Y Y^{\dagger}\right)_{i i} v_{\mathrm{w}}^{2}}{2 M_{i}}, \quad m_{\star} \equiv 4 \pi v_{\mathrm{w}}^{2} \sqrt{\frac{8 \pi^{3} g_{*}}{90 M_{\mathrm{P}}^{2}}}
$$

and the Hubble rate is given by

$$
H(T) \equiv T^{2} \sqrt{\frac{8 \pi^{3} g_{*}}{90 M_{\mathrm{P}}^{2}}}, \quad H_{i} \equiv H\left(T=M_{i}\right) .
$$

The value of the lepton asymmetry is rather sensitive to the value of $\epsilon$, and the parameter $M_{0}$ is constrained to be relatively small, $M_{0} \lesssim 3 \mathrm{TeV}$ (see Fig. 1), from the Higgs mass requirement. In Fig. 4 we show the resulting abundances of the two neutrino species obtained from the Boltzmann equations together with the corresponding equilibrium densities, and the net lepton abundance. To obtain the observed BAU, the net lepton abundance must be $\eta_{L} \equiv Y_{L-\bar{L}}=1.1 \times 10^{-9}$. The cross sections of the relevant processes are given in Appendix B. A benchmark set of parameters (case 1) leading to the desired value is given by $\epsilon=2.3 \times 10^{-4},\left|\alpha_{0}\right|=1.23 \times 10^{-3}$ and $M_{0}=0.4 \mathrm{TeV}$. The mass of the heavier neutrino is fixed by the vacuum

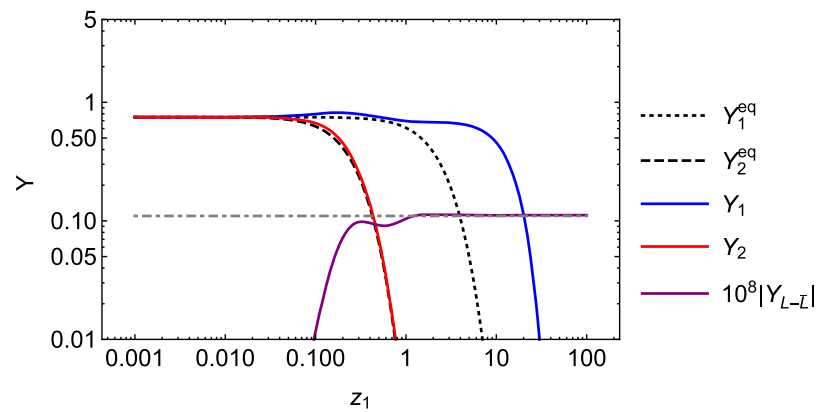

FIG. 4. The benchmark scenario (case 1) in Table I with mass hierarchy $M_{2}=79.5 \mathrm{TeV}$ and $M_{1}=8.6 \mathrm{TeV}$. Shown in the figure are the abundances of the two neutrinos with solid blue and red lines. The dashed lines show the corresponding equilibrium densities. The purple curve shows the resulting net lepton abundance normalized by a factor $10^{-8}$ so that the value corresponding to the observed $\mathrm{BAU}$ is $Y_{L-\bar{L}}=0.11 \times 10^{-8}$ shown by the horizontal dotted-dashed line. 


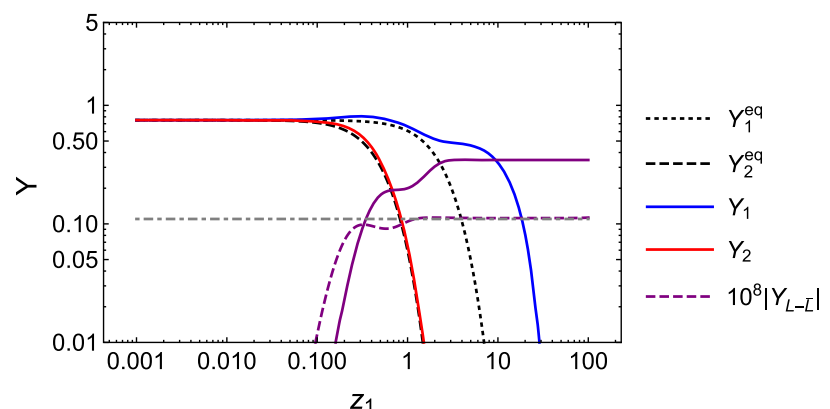

(a) $\epsilon \rightarrow 2 \epsilon$

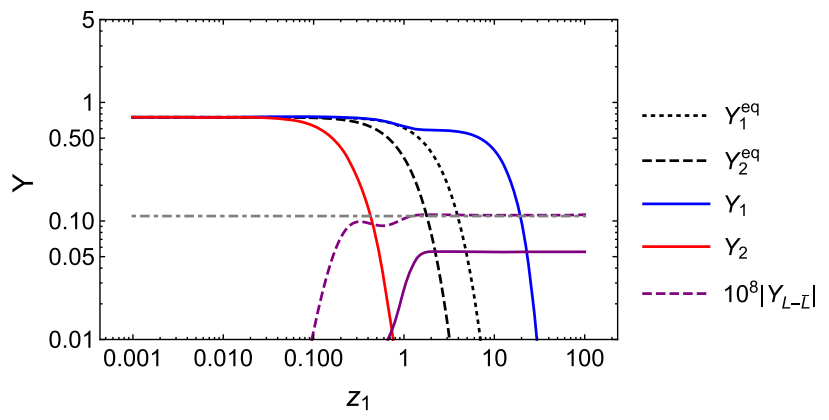

(b) $K_{2} \rightarrow 10 K_{2}$

FIG. 5. We show in these plots a comparison of the main results presented in Fig. 4 (dashed purple curve) and cases 2 (upper panel) and 3 (lower panel) presented in Table I. See the text for more details.

alignment to $79.5 \mathrm{TeV}$, while with the above parameters the mass of the lighter one becomes $8.6 \mathrm{TeV}$. The values of the light-neutrino Yukawa couplings, $Y$, enter the analysis via factors $K_{1}=0.01, K_{2}=4.0$. The scalar self-coupling enters through the trilinear coupling $\beta \varphi\left(H^{\dagger} H\right), \beta=$ $2.5 \times 10^{-4} \mathrm{TeV}$. Finally, with these parameters the mass of the singlet scalar is $M_{\varphi}=3.0 \mathrm{TeV}$.

This result is clearly sensitive to the values of the parameters involved in the analysis. We have checked that changing the parameter $M_{0}$ by a factor of two has hardly any effect for the asymmetry. This is expected, as $M_{0}$ does not affect the amount of $C P$ violation. On the other hand, the effects of varying $\epsilon$ in Eq. (10) and the light-neutrino mass parameters entering via $K_{2}$ have big effects on the resulting lepton asymmetry. For concreteness, we show in Fig. 5 the results considering $\epsilon \rightarrow 2 \epsilon$ (case 2) and $K_{2} \rightarrow$ $10 K_{2}$ (case 3). In Table I we list the values of the benchmark point (case 1) and the numerical values considered in cases 2 and 3 . In the second case the final lepton asymmetry is enhanced, while in the third case it is suppressed. For comparison, the dashed purple line shows our benchmark point of Fig. 4.

From the numerical results we see that the effect due to changing $\epsilon$ can be compensated for by changing $K_{2}$ accordingly. As a consequence, one may expect that a sizable portion of parameter space exists, within which the observed BAU can be produced by this model.
TABLE I. We show the values of the model parameters, $K_{2}, \alpha_{0}$, $\epsilon, \beta$, and the values of $v$ and $M_{1}$ for the cases 1-3 (see the text for details). All masses and $v$ are in TeV. The heavy-neutrino masses, $M_{1}$ and $M_{2}$, are determined by the vacuum and the matrix $\alpha$. For all the cases we use the same following benchmark values of the remaining parameters: $K_{1}=0.01, M_{0}=0.4 \mathrm{TeV}$, and $M_{\varphi}=3.0 \mathrm{TeV}$. The mass of the heavier neutrino is the same, $M_{2}=79.5 \mathrm{TeV}$, for all the cases.

\begin{tabular}{lcccccr}
\hline \hline No. & $K_{2}$ & $10^{4}\left|\alpha_{0}\right|$ & $10^{4} \epsilon$ & $10^{4} \beta$ & $10^{-4} v$ & $M_{1}$ \\
\hline 1 & 4.0 & 12.3 & 2.3 & 2.5 & 3.5 & 8.6 \\
2 & 4.0 & 13.5 & 4.6 & 2.5 & 3.5 & 16.7 \\
3 & 40 & 12.3 & 2.3 & 2.5 & 3.5 & 8.6 \\
\hline \hline
\end{tabular}

\section{CONCLUSIONS}

In this paper we have considered an extension of the SM where the Higgs boson arises as a pseudo-NambuGoldstone boson. As a concrete framework, we have considered the $\mathrm{SO}(5) / \mathrm{SO}(4)$ coset in terms of elementary scalar fields. The scalar content of the theory is accommodated in the fundamental of $\mathrm{SO}(5)$, and in addition to the SM Higgs sector it contains a real singlet scalar field. Furthermore, we added two heavy RH neutrinos to explain the masses and mixings of the light neutrinos and also to generate BAU via leptogenesis. The neutrino Majorana masses are generated through the interplay between a bare mass term and a mass term arising dynamically from the vacuum expectation value of the scalar singlet.

We found that the vacuum structure and the mass of the Higgs boson imply stringent bounds on the model parameters. In particular, we demonstrated that these lead to the requirement that the heavier of the two $\mathrm{RH}$ neutrino mass eigenvalues is $\sim 80 \mathrm{TeV}$.

This in turn has consequences for the generation of the BAU via leptogenesis. As we have discussed, this depends sensitively on the assumed mass patterns of the RH neutrinos. We analyzed in detail the cases of degenerate and hierarchical RH neutrinos, and identified examples of viable values of the parameters.

One could extend the model by adding a third $\mathrm{RH}$ neutrino as well. This would be a suitable candidate for dark matter given a vanishing mixing of this state with the two other RH neutrinos. The vacuum analysis would not be affected by its presence as we have shown that the vacuum alignment is determined only by the heaviest $\mathrm{RH}$ neutrino states. Furthermore, our analysis of BAU would also remain unaffected.

\section{ACKNOWLEDGMENTS}

This work has been supported by the Academy of Finland, Grant No. 267842. The CP3-Origins center is partially funded by the Danish National Research Foundation, Grant No. DNRF90. T. A. acknowledges partial funding from a Villum foundation grant. 


\section{APPENDIX A: DEFINITIONS}

\section{Definitions for resonant leptogenesis}

To estimate the $B-L$ asymmetry, we follow Refs. [27,33,37]. The final asymmetry is given as a sum of the contributions from the two lightest RH neutrinos, and three lepton flavors

$$
N_{B-L}^{\mathrm{f}}=\sum_{i, \alpha} N_{\Delta_{\alpha}}^{i}=\sum_{i, \alpha} \epsilon_{i \alpha} \kappa\left(K_{1 \alpha}+K_{2 \alpha}\right),
$$

where $i=1,2, \alpha=e, \mu, \tau$, and we define

$\epsilon_{i \alpha} \equiv-\frac{\Gamma_{i \alpha}-\bar{\Gamma}_{i \alpha}}{\sum_{\alpha}\left(\Gamma_{i \alpha}+\bar{\Gamma}_{i \alpha}\right)}, \quad K_{i \alpha} \equiv \frac{\Gamma_{i \alpha}+\bar{\Gamma}_{i \alpha}}{H\left(T=M_{i}\right)}=\frac{\left|m_{D \alpha i}\right|^{2}}{M_{i} m_{\star}}$,

and

$$
\begin{aligned}
\kappa(x) \equiv & \frac{2}{x z_{B}(x)}\left[1-\exp \left(-\frac{x z_{B}(x)}{2}\right)\right], \\
& \text { where } z_{B}(x) \simeq 2+4 x^{0.13} \mathrm{e}^{-\frac{2.5}{x}} .
\end{aligned}
$$

In the degenerate limit, the final asymmetry can be written as

$N_{B-L}^{\mathrm{f}} \simeq \frac{\bar{\epsilon}\left(M_{1}\right)}{3 \delta_{2}}\left(\frac{1}{\bar{K}_{1}}+\frac{1}{\bar{K}_{2}}\right) \sum_{\alpha} \kappa\left(K_{1 \alpha}+K_{2 \alpha}\right)\left(\mathcal{I}_{12}^{\alpha}+\mathcal{J}_{12}^{\alpha}\right)$,

where

$$
\begin{aligned}
& \mathcal{I}_{i j}^{\alpha} \equiv \frac{\operatorname{Im}\left[m_{D \alpha i}^{*} m_{D \alpha j}\left(m_{D}^{\dagger} m_{D}\right)_{i j}\right]}{M_{i} M_{j} m_{\mathrm{atm}} m_{\star}}, \\
& \mathcal{J}_{i j}^{\alpha} \equiv \frac{\operatorname{Im}\left[m_{D \alpha i}^{*} m_{D \alpha j}\left(m_{D}^{\dagger} m_{D}\right)_{j i}\right.}{M_{i} M_{j} m_{\mathrm{atm}} m_{\star}} \frac{M_{i}}{M_{j}},
\end{aligned}
$$

and

$$
\bar{K}_{i} \equiv \sum_{\alpha} K_{i \alpha}, \quad i=1,2
$$

Assuming that the baryon-to-photon ratio at recombination satisfies $\eta_{B} \simeq 0.01 N_{B_{L}}^{f}$, for normal hierarchy it is found that

$$
\begin{aligned}
\delta_{2} & \simeq 0.01 \frac{\bar{\epsilon}\left(M_{1}\right)}{\eta_{B}} f\left(m_{\nu}, \Omega\right) \\
& \simeq 0.8 \times 10^{-5}\left(\frac{f\left(m_{\nu}, \Omega\right)}{f_{\max }}\right)\left(\frac{M_{1}}{10^{6} \mathrm{GeV}}\right),
\end{aligned}
$$

where $f_{\max } \simeq 0.005$, and the function $f$ incorporates the dependence on the light-neutrino masses and mixings: $f\left(m_{\nu}, \Omega\right) \equiv \frac{1}{3}\left(\frac{1}{\bar{K}_{1}}+\frac{1}{\bar{K}_{2}}\right) \sum_{\alpha} \kappa\left(K_{1 \alpha}+K_{2 \alpha}\right)\left(\mathcal{I}_{12}^{\alpha}+\mathcal{J}_{12}^{\alpha}\right)$.

\section{Definitions for the hierarchical case}

In this appendix we give the decay, scattering and washout functions that appear in the Boltzmann equations, Eq. (21). We follow the notations of Ref. [32]. We define

$$
W_{1 D_{i}} \equiv \frac{1}{2 Y_{L}^{\mathrm{eq}}} D_{i} \quad \text { where } D_{i} \equiv \frac{\gamma_{D_{i}}^{\mathrm{eq}}}{n_{\gamma}^{\mathrm{eq}} H}=K_{i} z_{i}^{2} Y_{i}^{\mathrm{eq}} \frac{K_{1}\left(z_{i}\right)}{K_{2}\left(z_{i}\right)},
$$

$$
D_{21} \equiv K_{21} z_{2}^{2} Y_{2}^{\mathrm{eq}} \frac{K_{1}\left(z_{2}\right)}{K_{2}\left(z_{2}\right)}
$$

The scattering function for the process $i j \rightarrow m n$ reads

$$
\begin{aligned}
S_{i j \rightarrow m n} \equiv & \frac{\gamma_{i j \rightarrow m n}^{\mathrm{eq}}}{n_{\gamma}^{\mathrm{eq}} H}=\frac{m_{i}}{g_{\gamma} H_{i}} \frac{1}{32 \pi^{2}} z_{i} \\
& \times \int_{w_{\min }}^{\infty} \mathrm{d} w \sqrt{w} K_{1}(\sqrt{w}) \hat{\sigma}_{i j \rightarrow m n}\left(w \frac{m_{i}^{2}}{z_{i}^{2}}\right), \\
w_{\min } & =\max \left\{\left(m_{i}+m_{j}\right)^{2},\left(m_{m}+m_{n}\right)^{2}\right\} .
\end{aligned}
$$

We define

$$
\begin{aligned}
W_{S_{1}}= & \frac{1}{Y_{L}^{\mathrm{eq}}}\left(2 S_{N_{1} t \rightarrow L Q}+S_{N_{1} H \rightarrow L \varphi}+S_{N_{1} \varphi \rightarrow L H}\right) \\
& +\frac{Y_{1}}{Y_{L}^{\mathrm{eq}} Y_{1}^{\mathrm{eq}}}\left(S_{N_{1} L \rightarrow Q t}+S_{N_{1} L \rightarrow H \varphi}\right), \\
W_{S_{2}}= & \frac{1}{Y_{L}^{\mathrm{eq}}}\left(2 S_{N_{2} t \rightarrow L Q}+S_{N_{2} H \rightarrow L \varphi}+S_{N_{2} S_{\rightarrow}^{1} \rightarrow H}+S_{N_{2} S^{2} \rightarrow L H}\right) \\
& +\frac{Y_{2}}{Y_{L}^{\mathrm{eq}} Y_{2}^{\mathrm{eq}}}\left(S_{N_{2} L \rightarrow Q t}+S_{N_{2} L \rightarrow H \varphi}\right),
\end{aligned}
$$

and

$$
\begin{aligned}
S_{1}= & 2 S_{N_{1} L \rightarrow Q t}+4 S_{N_{1} Q \rightarrow L t}+2 S_{N_{1} L \rightarrow H \varphi}+4 S_{N_{1} H \rightarrow L \varphi} \\
& +2 S_{N_{1} \varphi \rightarrow L H} \\
S_{2}= & 2 S_{N_{2} L \rightarrow Q t}+4 S_{N_{2} Q \rightarrow L t}+2 S_{N_{2} L \rightarrow H \varphi}+4 S_{N_{2} H \rightarrow L \varphi} \\
& +2 S_{N_{2} \varphi \rightarrow L H}+2 S_{N_{2} \varphi \stackrel{2}{\rightarrow} L H} .
\end{aligned}
$$

\section{APPENDIX B: CROSS SECTIONS}

All the cross sections are summed over both the initial and final state degrees of freedoms (gauge, spins, lepton 
flavor). In some of the formulas we have found slightly different coefficients compared to [32].

\section{Decay widths}

$$
\begin{aligned}
\Gamma_{i} \equiv & \Gamma\left(N_{i} \rightarrow L H\right)+\Gamma\left(N_{i} \rightarrow \overline{L H}\right)=\frac{\left(Y Y^{\dagger}\right)_{i i} M_{i}}{8 \pi} . \\
\Gamma_{j i} \equiv & \Gamma\left(N_{j} \rightarrow N_{i} \varphi\right) \\
= & \frac{\left|\left(\alpha^{\dagger} \alpha\right)_{j i}\right|^{2} M_{j}}{16 \pi}\left[\left(1+\frac{M_{i}}{M_{j}}\right)^{2}-\frac{M_{\varphi}^{2}}{M_{j}^{2}}\right] \\
& \cdot \sqrt{\left(1-\frac{M_{i}^{2}}{M_{j}^{2}}-\frac{M_{\varphi}^{2}}{M_{j}^{2}}\right)^{2}-4 \frac{M_{i}^{2}}{M_{j}^{2}} \frac{M_{\varphi}^{2}}{M_{j}^{2}}} .
\end{aligned}
$$

\section{Scattering cross sections}

Below we tabulate the relevant cross sections $\sigma(i j \rightarrow m n)$. The reduced cross sections are obtained from these as

$$
\hat{\sigma}(i j \rightarrow m n)=\frac{1}{s} \delta\left(s, m_{i}^{2}, m_{j}^{2}\right) \sigma(i j \rightarrow m n),
$$

where

$$
\delta(a, b, c)=a^{2}+b^{2}+c^{2}-2 a b-2 a c-2 b c .
$$

a. $N_{i} L \rightarrow Q \bar{t}$

$\sigma\left(N_{i} L \rightarrow Q \bar{t}\right)=\frac{N_{c}\left(Y Y^{\dagger}\right)_{i i} y_{t}^{2}}{8 \pi} \frac{s}{\left(s-m_{h}^{2}\right)^{2}}$. b. $N_{i} Q \rightarrow L t$

$$
\begin{aligned}
\sigma\left(N_{i} Q \rightarrow L t\right)= & \frac{N_{c}\left(Y Y^{\dagger}\right)_{i i} y_{t}^{2}}{8 \pi\left(s-M_{i}\right)^{2}}\left[\frac{s-2 M_{i}^{2}+2 m_{h}^{2}}{s-M_{i}^{2}+m_{h}^{2}}\right. \\
& \left.+\frac{M_{i}^{2}-2 m_{h}^{2}}{s-M_{i}^{2}} \log \frac{s-M_{i}^{2}+m_{h}^{2}}{m_{h}^{2}}\right] .
\end{aligned}
$$

c. $N_{i} L \rightarrow H \varphi$

$$
\sigma\left(N_{i} L \rightarrow H \varphi\right)=\frac{\left(Y Y^{\dagger}\right)_{i i} \beta^{2}}{8 \pi} \frac{s-M_{\varphi}^{2}}{s^{3}} .
$$

d. $N_{i} \varphi \rightarrow L H$ via $N_{j}$

$$
\begin{aligned}
\sigma\left(N_{i} \varphi \stackrel{j}{\rightarrow} L H\right)= & \frac{\left(Y Y^{\dagger}\right)_{i i}\left|\left(\alpha \alpha^{\dagger}\right)_{j i}\right|^{2}}{16 \pi\left(s-M_{j}^{2}\right)^{2} \sqrt{\delta\left(s, M_{i}^{2}, M_{\varphi}^{2}\right)}} \\
& \times\left[\left(s+M_{i}^{2}-M_{\varphi}^{2}\right)\left(s+M_{j}^{2}\right)-4 M_{i} M_{j} s\right] .
\end{aligned}
$$

e. $N_{i} N_{j} \rightarrow H H$

$\sigma\left(N_{i} N_{j} \rightarrow H H\right)=\frac{\left|\left(\alpha \alpha^{\dagger}\right)_{j i}\right|^{2} \beta^{2}}{8 \pi} \frac{s-\left(M_{i}+M_{j}\right)^{2}}{\left(s-M_{\varphi}^{2}\right)^{2} \sqrt{\delta\left(s, M_{i}^{2}, M_{j}^{2}\right)}}$.

f. $N_{i} H \rightarrow L \varphi$

$$
\begin{aligned}
\sigma\left(N_{i} H \rightarrow L \varphi\right)= & \frac{\left(Y Y^{\dagger}\right)_{i i} \beta^{2}}{16 \pi\left(s-M_{i}^{2}\right)^{2}} \\
& \times \log \frac{s^{2}\left(s-M_{i}^{2}-M_{\varphi}^{2}\right)^{2}+s^{2} \mathcal{E}_{i}^{2}}{M_{i}^{4} M_{\varphi}^{4}+s^{2} \mathcal{E}_{i}^{2}} .
\end{aligned}
$$

[1] P. Minkowski, Phys. Lett. 67B, 421 (1977).

[2] M. Gell-Mann, P. Ramond, and R. Slansky, Supergravity, edited by F. Nieuwenhuizen and D. Friedman (North Holland, Amsterdam, 1979), p. 315.

[3] T. Yanagida, Proc. of the Workshop on Unified Theories and the Baryon Number of the Universe O. Sawada and A. Sugamoto (National Laboratory High Energy Physics, Tsukuba, Japan, 1979).

[4] R. N. Mohapatra and G. Senjanovic, Phys. Rev. Lett. 44, 912 (1980).

[5] J. Schechter and J. W. F. Valle, Phys. Rev. D 22, 2227 (1980).

[6] J. Schechter and J. W. F. Valle, Phys. Rev. D 25, 774 (1982).

[7] V. A. Kuzmin, V. A. Rubakov, and M. E. Shaposhnikov, Phys. Lett. 155B, 36 (1985)

[8] M. Fukugita and T. Yanagida, Phys. Lett. 174B, 45 (1986).
[9] M. Schmaltz and D. Tucker-Smith, Annu. Rev. Nucl. Part. Sci. 55, 229 (2005).

[10] S. R. Coleman and E. J. Weinberg, Phys. Rev. D 7, 1888 (1973).

[11] T. Alanne, H. Gertov, A. Meroni, and F. Sannino, Phys. Rev. D 94, 075015 (2016).

[12] G. M. Pruna and T. Robens, Phys. Rev. D 88, 115012 (2013).

[13] C. Englert, T. Plehn, D. Zerwas, and P. M. Zerwas, Phys. Lett. B 703, 298 (2011).

[14] B. Batell, S. Gori, and L.-T. Wang, J. High Energy Phys. 06 (2012) 172.

[15] D. Buttazzo, F. Sala, and A. Tesi, J. High Energy Phys. 11 (2015) 158.

[16] T. Robens and T. Stefaniak, Eur. Phys. J. C 75, 104 (2015). 
[17] T. Robens and T. Stefaniak, Eur. Phys. J. C 76, 268 (2016).

[18] J. McDonald, Phys. Rev. D 50, 3637 (1994).

[19] J. M. Cline and K. Kainulainen, J. Cosmol. Astropart. Phys. 01 (2013) 012.

[20] T. Alanne, K. Tuominen, and V. Vaskonen, Nucl. Phys. B889, 692 (2014).

[21] J. A. Casas, J. R. Espinosa, M. Quiros, and A. Riotto, Nucl. Phys. B436, 3 (1995); Nucl. Phys. B439, 466(E) (1995).

[22] M. Gonderinger, Y. Li, H. Patel, and M. J. Ramsey-Musolf, J. High Energy Phys. 01 (2010) 053.

[23] P. A. R. Ade et al. (Planck Collaboration), Astron. Astrophys. 594, A13 (2016).

[24] A. Riotto and M. Trodden, Annu. Rev. Nucl. Part. Sci. 49, 35 (1999).

[25] T. Asaka, S. Blanchet, and M. Shaposhnikov, Phys. Lett. B 631, 151 (2005).

[26] T. Asaka and M. Shaposhnikov, Phys. Lett. B 620, 17 (2005).
[27] P. Di Bari, P. O. Ludl, and S. Palomares-Ruiz, J. Cosmol. Astropart. Phys. 11 (2016) 044.

[28] J. A. Casas and A. Ibarra, Nucl. Phys. B618, 171 (2001).

[29] S. Davidson and A. Ibarra, Phys. Lett. B 535, 25 (2002).

[30] A. Pilaftsis, Phys. Rev. D 56, 5431 (1997).

[31] A. Pilaftsis and T. E. J. Underwood, Nucl. Phys. B692, 303 (2004).

[32] M. Le Dall and A. Ritz, Phys. Rev. D 90, 096002 (2014).

[33] S. Pascoli, S. T. Petcov, and A. Riotto, Nucl. Phys. B774, 1 (2007).

[34] M. Garny, A. Kartavtsev, and A. Hohenegger, Ann. Phys. (Amsterdam) 328, 26 (2013).

[35] P. S. B. Dev, P. Millington, A. Pilaftsis, and D. Teresi, Nucl. Phys. B891, 128 (2015).

[36] M. Drewes, B. Garbrecht, D. Gueter, and J. Klaric, J. High Energy Phys. 12 (2016) 150.

[37] S. Pascoli, S. T. Petcov, and A. Riotto, Phys. Rev. D 75, 083511 (2007).

[38] W. Buchmuller, P. Di Bari, and M. Plumacher, New J. Phys. 6, 105 (2004). 